\title{
Statistical analysis of the learning attitudes of undergraduates to major courses
}

\author{
Qingtian Zhang ${ }^{a}$ and Guikun $\mathrm{Hu}^{\mathrm{b}}$ \\ College of Marine and Environmental Sciences, Tianjin University of Science and Technology, \\ Tianjin 300457, China \\ aqtzhang@163.com, bhuguikun@163.com
}

\begin{abstract}
The learning attitude of college students to the professional curriculum determines the success or failure of higher education to some degree. A survey on learning attitude of junior to major courses was carried out among four classes, and then multivariate statistical analysis was conducted for the survey data, in order to provide scientific guidance for undergraduate education. The attitude of junior to the professional curriculum involved many aspects, and it had real impact on the efficiency of learning. Learning attitude involved many aspects, while the listening attitude in class and self-learning attitude was still the most important aspect. Teachers having different responsibilities should cooperate to complete the teaching task well. The understanding of the education internationalization is becoming a part of learning attitude; professional teachers should guide students to meet the needs of new international markets.
\end{abstract}

Keywords: Higher education; major course; learning attitude; internationalization; statistical analysis.

\section{本科生专业课程学习态度的统计分析}

\author{
张青田, 胡桂坤 \\ 天津科技大学 海洋与环境学院, 天津, 300457 中国
}

摘 要: 大学生对专业课程的学习态度在很多程度上决定了高等教育的成败。运用多元统计分 析的方法处理大学生学习态度的调查数据, 分析影响学习的关键因素, 以期为本科生教学提 供科学指导。学生对专业课程的态度涉及多个方面, 并切实影响着学习的效果。听课态度及 自主学习态度是影响学习的最重要环节; 不同职责的教师应该协同合作完成教学任务。对教 育国际化的认识正成为学习态度的组成部分, 专业教师应该引导学生认识新市场的专业需求。

关键词：高等教育；专业课程；学习态度；国际化；统计分析.

\section{1. 前言}

学习态度是大学生对学习及相关情境表现出来的相对稳定的心理倾向, 它是后天社会化作用 的产物, 受主体内、外多种因素的制约。学习态度的对象包括课程、教师、纪律以及学习前途 等很多方面, 学习态度直接影响着个体的学习状态和学习效率 ${ }^{[1-3]}$ 。

专业课程学习是大学学习不同于其他学习阶段的重要特征, 因为绝大多数毕业生要走上工作 岗位, 接受社会对专业课程学习的考验。在很大程度上, 专业课程的学习优劣直接决定了学 生的就业前景和教育的成败。现今, 高等教育的国际化已是大势所趋 ${ }^{[4]}$, 它涉及国家的利益 和国际竞争力, 无论发达国家还是发展中国家都无法回避。教育国际化形势下, 大学教育的 目的之一是要培养适应国际化潮流的毕业生, 在职业技能和思维方面满足市场的需求 ${ }^{[5-6]}$ 。大 学生的竞争是国际性的竞争, 专业课程的学习面临着更高的要求。研究表明对学习及专业的 态度不仅影响大学生的学习成绩, 而且对其身心健康也产生了一定的影响 ${ }^{[7]}$ 。在新形势下开展 大学生对专业课程的认知态度是非常必要的。 
国内关于大学生学习态度的研究较多, 但多数局限于各种态度的比例分析, 缺乏统计学的探 讨和综合影响的分析。本研究对海洋专业的大三学生进行了专业课程的学习态度调查, 希望 了解学生对专业课程的态度, 通过多元统计方法分析影响学习的关键因素, 为新形势下的本 科生教学提供科学的参考; 把握学生在不同学习环节的关键问题, 指导学生克服困难, 提高 成绩，适应专业培养的新需求。

\section{2. 研究和统计分析方法}

\section{1 . 调查背景介绍}

以问卷形式搜集本科生学习态度的数据, 问卷采用匿名形式进行, 采集专业班级和性别信息。 在学习态度方面共设计了 3 大类项目, 包括对待专业课的态度 (课上听讲及课前、课后的自 主学习等), 毕业计划 (企业类型和出国留学等), 以及对教育国际化的认知态度（本专业 在国际上地位，对国际交换生的态度等）。

选择本科 3 年级的两个理科专业共四个班级进行了摸底调查, 共发放问卷 105 张, 有效问卷 100 份。其中男生 54 人, 女生 46 人。舍弃低年级学生是因为其学习态度不如高年级学生积极; 听从家长安排专业和服从调剂的比例较高, 对专业的了解很少 ${ }^{[1,8]}$ 。三年级学生已经开始了一 年多的专业课学习, 有了比较清醒的认识, 是适合研究的对象。同时统计了四个班级在 3 年 内获得奖学金的情况作为分析参考。

2.2. 多元统计分析

将问卷中不同态度选项作为班级、性别的学习属性建立数据库, 进行多维标度 (MDS) 分析 和关键因素 (BEST) 分析, 统计和绘图借助 PRIMER $®$ 软件完成。多维标度法是一种将多维 空间的研究对象 (样本或变量) 简化到低维空间进行定位和归类, 同时又保留对象间原始关 系的数据分析方法。不同班级男女生数量有差异, 对数据进行了标准化处理, 然后计算对象 间的欧氏距离(Euclidean distance) $d_{j k}$, 再利用 MDS 分析各种态度的综合差异。

$$
d_{j k}=\sqrt{\sum_{i=1}^{p}\left(y_{i j}-y_{i k}\right)^{2}}
$$

式中, $j, k$ 为要比较的班级和性别的分类组合; $i=1,2 \ldots p$, 为调查内容的条目, 即对不同问题 的态度。为了考量 MDS 结果的可信度, 用 Stress 参数测量 MDS 结果的回归适合度 ${ }^{[9]}$ 。

$$
\text { Stress }=\sqrt{\sum_{j} \sum_{k}\left(d_{j k}-\hat{d}_{j k}\right)^{2} / \sum_{j} \sum_{k} d_{j k}^{2}}
$$

式中, $\hat{d}_{j k}$ 是相应不相似性适合回归曲线预测的距离, 如果所有计算距离的 $d_{j k}=\hat{d}_{j k}$, 则 Stress 为 0 。 BEST 分析是对所有调查内容进行模拟排列, 找出影响结果的关键因素来简化问题。

\section{3. 结果分析和教学启示}

\section{1 结果分析讨论}

调查数据显示学生对专业课程学习的态度存在差异, 总体上呈现出多样化的态势。例如, 不 认真听课的原因多种多样, 不喜欢内容的占 30.3\%, 难以抗拒手机诱惑的占 $16.2 \%$, 还有 $13.1 \%$ 的学生做其他课程的作业等。限于篇幅, 重点分析这些差异在班级和性别间有何综合性影响, 不具体分析每项内容的差异。

图 1 展示了四个班级各种学习态度的综合差异, 图中数字编号第一位代表专业, 第二位代表 班级; 字母 $\mathrm{f}$ 代表女性, $\mathrm{m}$ 代表男性。图中各点间距离表示不同对象间态度的差异程度。由 图可知, 专业 1 男女学生的学习态度差异很大, 在图中相距很远, 尤其是专业 1 的 2 班男生 明显不同于其他学生。相对来说, 专业 2 的学生间态度差异较小, 但是 1 班男女生的差异很 大。总体上, 男生占据图的左上部, 而女生占据图的右下部。这说明学生对专业课程的学习 态度随着专业、性别而有差异; 这些差异可能与学生相互影响有关。 


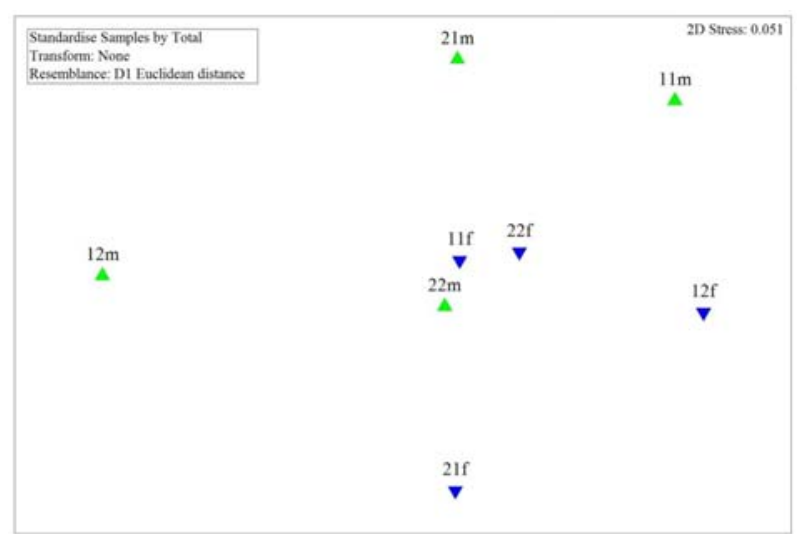

图1 四个班级各种学习态度的差异分析

为了分析和验证以上差异的影响, 分析了这些学生在1-3年级获得奖学金的情况（图2）。

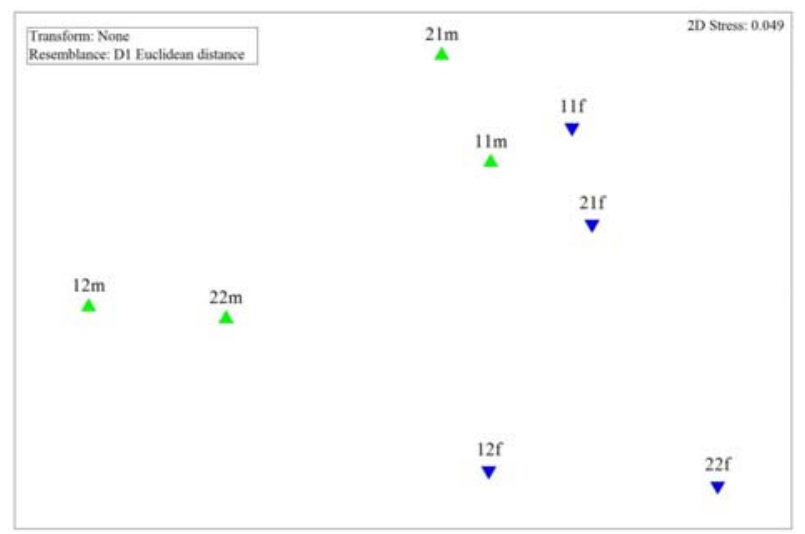

图2 四个班级获奖学金情况的差异分析

图 2 和图 1 的情况类似，性别间的差异更加明显。专业 1 的 2 班男生在 3 年间没有人获得奖 学金, 在图 1 和图 2 中都有明显表示。女生逃课较少, 获得奖学金较多也是很多学校的普遍 现象; MDS 图很好地展示了这些差别。两个 MDS 分析的 Stress 值都小于 0.1 , 说明结果有非 常好的解释意义 ${ }^{[9]}$ 。本调查对学生学习态度的分析内容能够反映学生学习的结果, 对分析学 习行为和指导学生有很好的参考价值。

在统计的所有条目中, BEST 分析选出 10 个因素作为反映学生学习态度的关键因素（相关系 数 $\mathrm{r}$ 为 0.95 ）。其中, 对待专业课程的学习态度占了 $60 \%$, 涉及课前预习, 课堂听讲和课后 作业等方面。将来的计划 (进入国际性企业, 出国留学等) 也是影响学习态度和成绩的重要 因素。对本专业在国际上的水平和国内外差异程度也影响着学生学习专业课程的态度。

3.2 教学启迪和要求

统计分析结果表明, 学生学习专业课程的态度影响着学习的效果, 对学校和教师也提出了新 的要求。首先, 授课情况和管理力度是决定学习态度的重要部分, 教师应该认真备课, 提高 学生学习兴趣, 如何吸引学生注意力是每位老师必须思考的问题。大学与中小学不同, 对于 课前和课后的监督较少, 一些学生就产生了懒惰心理。教师应该通过课上提问和批阅作业等 形式及时了解和督促学生做好自主学习工作。无论哪个学习阶段, 听课态度及其前后的自主 学习态度都是影响学习效果的最重要内容, 是教学过程中必须认真对待的。其次, 分析结果 也表明教学过程包括多个环节, 仅凭专业课教师来督促学习是远远不够的, 应该由多种职责 的教师分工合作; 鼓励和吸引不同职能的教师参与到学生管理中 [10]。以教师为主导, 大学生 为主体, 专业教师协助大学生制定学习目标和计划; 辅导员和任课教师协助制定个性化培养 方案, 在实施中进行解惑和监督 ${ }^{[3]}$ 。还有, 学生对就业和前途是关注的, 这影响着学习效果; 一些研究也表明认真学习的动力多数来自就业压力 ${ }^{[1]}$ 。这要求专业课教师应该与时俱进, 积 极了解专业前沿和国际形势, 以满足学生的需求, 这也是提高学习兴趣的一个方式。 


\section{4. 小结}

分析结果表明, 学生对专业课程的态度涉及多个方面, 并且切实影响着学习效果。课堂听课 效果及自主学习情况仍是影响学习的最重要环节。同时, 学习态度涉及多个方面, 不同职责 的教师应该协同合作完成教学任务。对教育国际化的认识已经成为学习态度的组成部分, 课 程中应该引导学生认识新市场的专业需求。

\section{致谢}

基金项目：天津市教育科学 “十二五” 规划课题（编号 HEYP6018）

\section{References}

[1] Zhao DL. An inquisition of university students' study attitude- lack of internal study motive and the counterplan [J]. Journal of Chengdu University (Education science edition). Vol. 22(2008), No.5, p.24-27.

[2] Duan XD. Investigation and analysis on the learning attitude of students in local normal colleges and universities- a case study of a normal college [J]. Science \& technology information. Vol (2012), No.23, p.24-25.

[3] Zou WM. A survey of learning attitudes of students in higher vocational colleges [J]. Theory and Practice of Education. Vol.34(2014), No.9, p.34-35.

[4]Sekiwu D. From localization to internationalization of higher education: Globalization and transformation of university education in Uganda [OL]. http://eric.ed.gov/?id=ED512372. 2010.

[5] Tabulawa R. Global influences and local responses: The restructuring of the University of Botswana, 1990-2000 [J]. Higher Education. Vol. (2007), No.53, p.457-482.

[6] Currie J. Globalisation Practices and the Professoriate in Anglo-Pacific and North American Universities [J]. Comparative Education Review. Vol.42(2008),No.1,p.15-29.

[7] Wang L, Ye MZ, Xiao XQ, et al. An investigation on the profession and learning attitude of students in medical university[J]. Chinese journal of school doctor. Vol.17(2003), No.1, p.39-40.

[8] Chen LS,Wu XY. Investigation and analysis on the learning attitude of students in colleges and universities - a case study in Shenyang universities[J]. Higher agriculture education. Vol.(2011), No.3, p.85-87.

[9]Clarke KR. 1993. Non-parametric multivariate analyses of changes in community structure [J]. Australian journal of ecology.Vol.18(1993),p.117-143.

[10] Hu GK, Zhang QT. Discussion on how to guide the freshmen to adapt the new university environment[J]. New west. Vol.(2014), No.2, p.100. 